\title{
Review on testosterone delivery by natural and synthetic nanoparticles
}

\begin{abstract}
The loading efficacies of testosterone with polyamidoamine PAMAN-G3 and PAMAM-G4 and chitosan- 15 and chitosan- $100 \mathrm{kDa}$ nanoparticles were compared in aqueous solution at $\mathrm{pH}$ 7.4. The results of multiple spectroscopic methods, transmission electron microscopy (TEM) and molecular modeling were used to characterize the testosterone binding process to polymer nanoparticles. Structural analysis showed testosterone-polymer bindings occur via hydrophobic, H-bonding contacts. The binding affinity is testosterone-chitosan $>$ testosterone-PAMAM. Transmission electron microscopy showed significant changes in carrier morphology with major changes in the diameter of the polymer aggregates as steroid loading occurred. Chitosan nanoparticles are more effective carriers than PAMAM dendrimers.
\end{abstract}

Keywords: Testosterone, Chitosan, Dendrimer, Delivery, TEM, Modeling, Synthetic polymers, Dendrimers, A Family of cationic polymers, Filter paper, Surface groups, Water solubility, Drug delivery, Spectroscopic, Biological properties, Intensity

Volume 5 Issue 2 - 2017

P Chanphai, Berube G, Tajmir Riahi HA

Department of Chemistry-Biochemistry and Physics, University of Quebec at Trois Rivieres, Canada

Correspondence: HA Tajmir Riahi, Department of ChemistryBiochemistry and Physics, C. P. 500, Trois Rivieres (Quebec), G9A 5H7, Canada, Tel 819-376-50II (3326) Email heidar.alitajmir-riahi@uqtr.ca

Received: January 12, 2017 | Published: March 09, 2017
Abbreviations: Ch, Chitosan; PAMAM, Poly(Amidoamine); FTIR, Fourier Transform Infrared; TEM, Transmission Electron Microscopy

\section{Introduction}

Natural and synthetic polymers are widely used as drug delivery tools in pharmaceutical and nanomedicine biotechnology. ${ }^{1-5}$ Among synthetic polymers, dendrimers, a family of cationic polymers, are promising nonviral vectors for gene and drug delivery because of a well-defined molecular shape, controlled chemical structure, high water solubility, large number of chemically versatile surface groups, and unique architecture. ${ }^{6-8}$ Natural cationic polymers such as chitosan are attractive candidates for therapeutic applications as they generally are non-toxic, being derived from renewable resources. ${ }^{9}$ Biodegradable, biocompatible and nontoxic chitosan nanoparticles are of a major interest in drug delivery systems. They form complexes with antibiotics, anticancer drugs and therapeutic proteins. ${ }^{10-12}$ Chitosan and its derivatives have the desired properties for safe use as pharmaceutical drug delivery tools. This has prompted accelerated research activities worldwide on chitosan nanoparticles as drug delivery tools..$^{13-16}$

Testosterone is the main androgenic hormone which controls many physiological processes such as, sexual functions and secondary sex characteristics, muscle protein metabolism, plasma lipid and bone metabolism. ${ }^{17}$ Testosterone can also be used as a natural template to construct semi-synthetic analogues such as dimers, testosterone amide derivatives, as well as testosterone-cytotoxic hybrid molecules. ${ }^{18-22}$ These novel semi-synthetic molecules can be tested for their biological properties, using diverse spectroscopic methods. ${ }^{18-25}$ Synthetic polymers are used as potential nanocarriers to deliver steroids in vitro and in vivo. ${ }^{26-28}$ Furthermore, chitosan and its derivatives were also tested as delivery tools for transporting steroids. ${ }^{29,30}$

In this review, the binding efficacies of testosterone with PAMAM dendrimers and chitosan nanoparticles were compared, using multiple spectroscopic, TEM images and molecular modeling results. Structural analysis regarding testosterone binding process and the effect of steroid-polymer conjugation on polymer morphology as well as the possibility of testosterone delivery by PAMAM and chitosan nanoparticles are discussed here.

\section{Experimental}

\section{Transmission electron microscopy}

The TEM images were taken using a Philips EM 208S microscope operating at $180 \mathrm{kV}$. The morphology of the testosterone conjugates with PAMAM dendrimers and chitosan nanoparticles in aqueous solution at $\mathrm{pH} 7.4$ were observed, using transmission electron microscopy. One drop $(5-10 \mu \mathrm{L})$ of the freshly-prepared mixture [polymer solution $(60 \mu \mathrm{M})+$ steroid solution $(60 \mu \mathrm{M})]$ in Tris- $\mathrm{HCl}$ buffer $\left(24 \pm 1{ }^{\circ} \mathrm{C}\right)$ was deposited onto a glow-discharged carboncoated electron microscopy grid. The excess liquid was absorbed by a piece of filter paper, and a drop of $2 \%$ uranyl acetate negative stain was added before drying at room temperature. ${ }^{28}$

\section{UV spectroscopy}

The UV-Vis spectra were recorded on a Perkin-Elmer Lambda spectrophotometer with a slit of $2 \mathrm{~nm}$ and scan speed of $400 \mathrm{~nm}$ min 1. Quartz cuvettes of $1 \mathrm{~cm}$ were used. The absorbance measurements were performed at $\mathrm{pH} 7.4$ by keeping the concentration of polymer constant $(60 \mu \mathrm{M})$, while increasing testosterone concentrations $(1 \mu \mathrm{M}$ to $60 \mu \mathrm{M})$. The binding constants of steroid-polymer conjugates were obtained according to the published method. ${ }^{31}$

\section{FTIR Spectroscopic measurements}

Infrared spectra were recorded on a FTIR spectrometer (Impact 420 model, Digilab), equipped with deuterated triglycine sulphate (DTGS) detector and $\mathrm{KBr}$ beam splitter, using $\mathrm{AgBr}$ windows. Solution of testosterone was added drop wise to the polymer solution with constant stirring to ensure the formation of homogeneous solution and to reach the target steroid concentrations of 15,30 and $60 \mu \mathrm{M}$ with a final polymer concentration of $60 \mu \mathrm{M}$. Spectra were collected after $2 \mathrm{~h}$ incubation of testosterone with polymer solution at room temperature, using hydrated films. Interferograms were accumulated over the spectral range 4000-600 $\mathrm{cm}^{-1}$ with a resolution of $2 \mathrm{~cm}^{-1}$ and 100 scans. The difference spectra [(polymer + steroid solution $)$ - 
(polymer solution)] were generated using water combination band at $2300 \mathrm{~cm}^{-1}$

\section{Molecular modeling}

The PAMAM-G4, chitosan and testosterone structures were generated using Chem Office Ultra 6.0 software suite. ${ }^{32,33}$ The drug was then automatically docked to the rough PAMAM-G4 and chitosan structures using ArgusLab 4.0.1 (ArgusLab 4.0.1, Mark A. Thompson, Planaria Software LLC, Seattle, WA, http:/www.arguslab.com). The docked testosterone-polymer structures were optimized by means of molecular dynamics, using the MM+ force field available in Hyper Chem Pro 7.0. The free binding energies of the optimized steroidpolymer complexes were calculated using the Ascore scoring function provided in the ArgusLab software..$^{13,14,28}$

\section{Results and discussion}

\section{TEM images and the morphology of testosterone- polymer aggregates}

TEM images showed major changes in the morphological of PAMAM dendrimers and chitosan nanoparticles upon conjugation with testosterone Figure 1. It can be seen that the free PAMAM-G3 and PAMAM-G4 Figure 1A \& 1B are spherical crystalline structures, with mean dimensions of $10 \pm 2 \mathrm{~nm},{ }^{34-41}$ while testosterone-PAMAM, present average dimensions of $19 \pm 3 \mathrm{~nm}$ (Figures 1A, 1B, 1E \& 1F). ${ }^{28}$ The results show that the steroid-PAMAM nanoparticles were larger in size than the free PAMAM due to testosterone encapsulation. This size differences could be attributed to expansion of the branches of PAMAM dendrimer to accommodate steroids within the hydrophobic core to reach high loading. It should be noted that the increase in particle size was more pronounce in the case of textosteronePAMAM-G4 than testosterone-PAMAM-G3 (Figures 1E \& 1F). PAMAM-G4 forms more stable conjugates than PAMAM-G3. Similar structural changes were observed for several drug-PAMAMconjugates. ${ }^{42,43}$

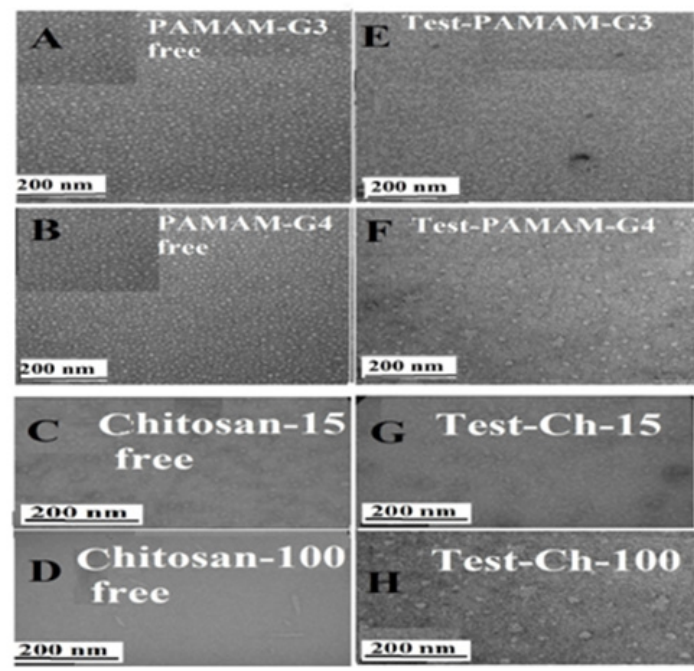

Figure I TEM photographs showing the morphology of the free PAMAM-G3 and PAMAM-G4 (A and B) and chitosan- 15 and chitosan- $100 \mathrm{kDa}(\mathrm{C}$ and $\mathrm{D})$ at $\mathrm{pH} 7.4$ and $24^{\circ} \mathrm{C}$ : in the presence of testosterone (E, F, G and $\left.\mathrm{H}\right)$.

The concentrations ofsteroid and polymer were $60 \mu \mathrm{M}$ in all samples.

The shapes of the free Ch-15 and Ch-100 kDa alongside with their testosterone conjugates are shown in the TEM images (Figures 1C, 1D,
$1 \mathrm{G} \& 1 \mathrm{H})$. TEM micrographs show that free chitosan had a markedly different shape depending on its size; Ch-15 has spherical-shaped, while Ch-100 is needle-shaped with smooth surface and narrow size distribution of about $90 \mathrm{~nm} .{ }^{44,45}$ Similar differences were observed for AFM images of Ch-15 and Ch-100 kDa where the result is attributed to the degree of polymer aggregation, as chitosan size increased. ${ }^{46}$ However, marked differences were observed in the morphology of the testosterone-chitosan aggregates. TEM images clearly showed the appearance of the aggregates of irregular shapes dispersed in solution when Ch-15 conjugates with testosterone (Figures $1 \mathrm{G}$ $\& 1 \mathrm{H})$. In addition, the bound $\mathrm{Ch}-100$ with testosterone showed major changes of the polymer morphological shape (Figure 1H). An increase of the spherical-shaped aggregates can be seen from TEM micrograph, suggesting that the elongated shapes were lost in favor of spherical-shaped in the testosterone-chitosan conjugates (Figures $1 \mathrm{G} \& 1 \mathrm{H})$. The loss of the elongated shape of chitosan nanoparticles after complex formation with testosterone is likely to be the result of the testosterone encapsulation. This is consistent with major particle size increase as encapsulation occurs (Figures $1 \mathrm{G} \& 1 \mathrm{H}$ ). Testosterone binding to chitosan, which is a linear polysaccharide that has multiple sites of interaction and therefore should be regarded as core-shell system with steroid (core) and chitosan (shell). ${ }^{47-51}$

\section{Stability and loading efficacy of testosterone-polymer conjugates by UV spectroscopy}

Steroid-polymer binding results in changes in the absorption spectra of the PAMAM and chitosan and the observed changes can be used to calculate the testosterone-polymer binding constants. ${ }^{28,31,52,53}$ Steroid-polymer complexation occurred with an increase in the intensity of polymer and testosterone absorption bands around $250 \mathrm{~nm}$ (Figure $2 \& 3$ ). The steroid-polymer binding constants were calculated as described, ${ }^{31}$ using plots of $1 /\left(\mathrm{A}-\mathrm{A}_{0}\right)$ vs (1/steroid concentrations) (Figures $2 \& 3$ ).

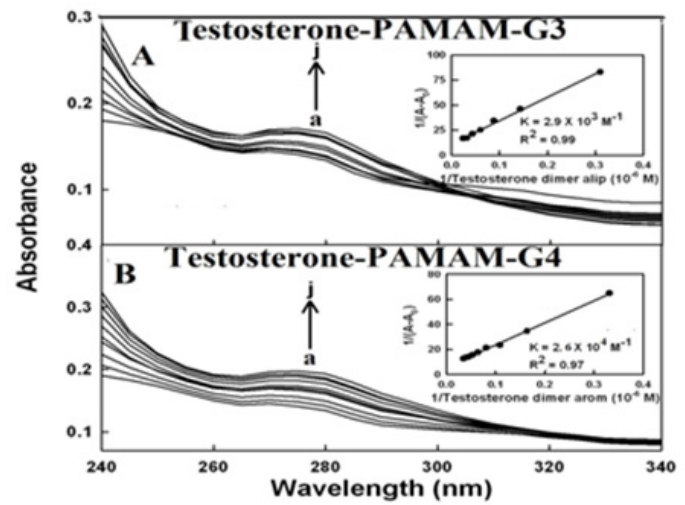

Figure 2 UV-visible spectra of PAMAM and its complexes with testosterone $(A)$ with free PAMAM-G3 and (B) PAMAM-G4 (a) at $60 \mu \mathrm{M}$ and testosterone (b-j) at I, 3, 5, I0, 20, 30, 40, 50 and $60 \mu \mathrm{M}$. Inset: plot of plot of I/(A-A $)$ vs (I/ steroid concentration) and binding constant $(K)$ for testosterone-PAMAM conjugates. $\mathrm{I} /\left(\mathrm{A}-\mathrm{A}_{0}\right)$.

The double reciprocal plot is linear and gives the overall binding constants for each complex, $\mathrm{K}_{\text {testosterone-PAMAM-G3 }}=2.9( \pm 0.6) \times 10^{3} \mathrm{M}^{-1}$, $\mathrm{K}_{\text {testosterone-PAMAM-G4 }}=2.6( \pm 0.3) \times 10^{4} \mathrm{M}^{-1}, \mathrm{~K}_{\text {testosterone-chitosan-15 }}=5.0( \pm 0.6)$ $\mathrm{x} 10^{4} \mathrm{M}^{-1}$ and $\mathrm{K}_{\text {testosterone-chitosan-100 }}=5.9( \pm 0.9) \times 10^{5} \mathrm{M}^{-1}$ Figure $2 \& 3$. The results showed that testosterone forms stronger adduct with chitosan nanoparticles than PAMAM dendrimers (Figures $2 \& 3$ ). The extra stability of acid-PAMAM-G4 conjugate is related to the presence of additional terminal charged $\mathrm{NH}_{2}$ groups (64 amino groups) compared to PAMAM-G3 (32 amino groups), that are involved in testosterone- 
polymer interactions. Similarly chitosan-100 kDa forms more stable steroid conjugates than chitosan- $15 \mathrm{kDa}$, due to the presence of more charged $\mathrm{NH}_{2}$ groups associated with larger chitosan nanoparticles.

The loading efficacy for testosterone-polymer conjugates was determined as reported. ${ }^{54}$

$\%$ Efficiency $=\frac{\text { Final absorption intensity }- \text { Initial absorption intensity }}{\text { Initial absorption intensity }} \times 100$

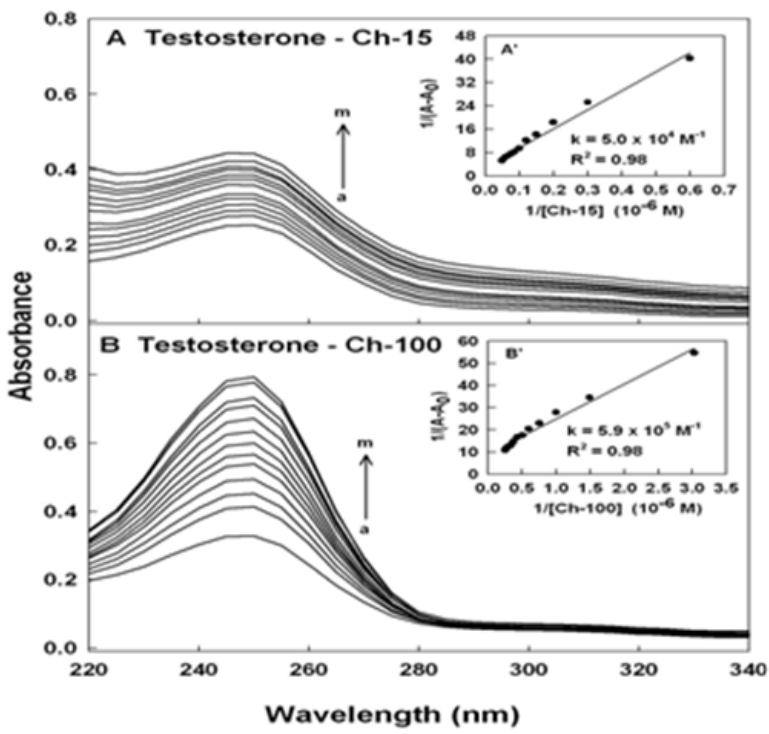

Figure 3 UV-visible spectra of chitosan and its complexes with testosterone (A) chitosan- 15 and (B) chitosan- $100 \mathrm{kDa}$ (a) with free testosterone at $60 \mu \mathrm{M}$ and chitosan (b-m) at I, 3, 5, I0, 20, 30, 40, 50, 60, 70, 80 and $90 \mu \mathrm{M}$. Inset: plot of $\mathrm{I} /\left(\mathrm{A}-\mathrm{A}_{0}\right) \mathrm{vs}$ ( $\mathrm{I} /$ chitosan concentration) and the binding constant $(\mathrm{K})$ for testosterone-chitosan conjugates.

The loading efficacy of testosterone conjugates estimated to be 30\% (PAMAM-G3), 40\% (PAMAM-G4), 45\% (chitosan-15) and $55 \%$ (chitosan-100 kDa) in these steroid-polymer conjugates.

\section{Binding analysis of testosterone-polymer conjugates by FTIR spectroscopy}

Testosterone-polymer interactions come from infrared results presented in Figure 4. Spectral shifting was observed for the polymer $\mathrm{C}=\mathrm{O}, \mathrm{C}-\mathrm{N}, \mathrm{C}-\mathrm{O}$ stretching and $\mathrm{OH}$ and $\mathrm{NH}$ bending, ${ }^{55,56}$ upon testosterone hydrophilic contacts with polymer polar groups. In the free PAMAM-G4 infrared spectrum, the bands at 1650 and $1558 \mathrm{~cm}^{-1}$ (OH and NH bending), 1471, 1380, 1303, 1159 and 1060 $\mathrm{cm}^{-1}$ (C-O and C-C stretch), exhibited shifting and intensity decreases, upon steroid-polymer complexation Figure 4A, complexes, 60 $\mu \mathrm{M}$. The observed spectral shifting was accompanied by a gradual decrease in the intensity of the above vibrational frequencies, in the difference spectra [(polymer solution + steroid solution $)-($ polymer solution)] of testosterone-polymer conjugates (Figure 4A, diffs 15 $\mu \mathrm{M})$. As testosterone concentrations increased to $60 \mathrm{mM}$, major decreases in the intensity of PAMAM vibrational frequencies were observed (Figure 4A, diffs $60 \mu \mathrm{M}$ ). The strong negative features in the difference spectra at 1648,1554 and $1051 \mathrm{~cm}^{-1}$ (testosteronePAMAM) are due to the loss of intensity of polymer major vibrational bands (Figure 4A, diffs $60 \mu \mathrm{M}$ ). The spectral changes observed are attributed to the hydrophilic interactions of steroid polar groups with polymer $\mathrm{OH}, \mathrm{NH}_{2}, \mathrm{C}-\mathrm{O}$ and $\mathrm{C}-\mathrm{N}$ groups. The hydrophilic interaction is more pronounced at high steroid concentrations (Figure 4A, diffs 60 $\mu \mathrm{M})$. Similarly, chitosan vibrational frequencies at 1638, 1530, 1379,
1151,1080 and $1035 \mathrm{~cm}^{-1}$ exhibited major changes upon testosterone conjugation (Figure 4B). The positive features observed at 1596$1594,1498-1488 \mathrm{~cm}^{-1}$ and negative features at 1073 and $1074 \mathrm{~cm}^{-1}$, in the difference spectra of testosterone-chitosan complexes are due to the participation of chitosan $\mathrm{OH}, \mathrm{NH}_{2}, \mathrm{C}-\mathrm{O}$ and $\mathrm{C}-\mathrm{N}$ groups in testosterone-polymer bindings (Figure 4B, diffs 15 and $60 \mu \mathrm{M}$ ).

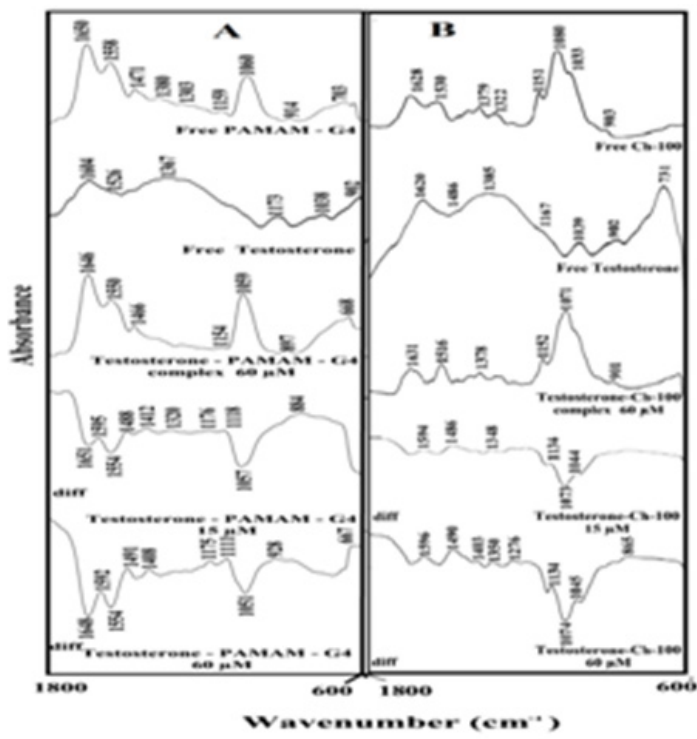

Figure 4 FTIR spectra in the region of $1800-600 \mathrm{~cm}^{-1}$ of hydrated films (pH 7.4) for free (A) PAMAM-G4 $(60 \mu \mathrm{M})$ and (B) chitosan- $100 \mathrm{kDa}(60 \mu \mathrm{M})$ and their testosterone complexes with difference spectra (diff.) (bottom two curves) and obtained at different testosterone concentrations (indicated on the figure).

\section{Docking study}

Docking experiments of testosterone to PAMAM-G4 and chitosan nanoparticles revealed the preferred binding sites as shown in Figure 5. The docking results showed testosterone is located in the PAMAM internal cavities surrounded by polymer hydrophobic groups with the free binding energy $-9.36 \mathrm{kcal} / \mathrm{mol}$ (Figure $5 \mathrm{~A}$ ), while testosterone is bound to the chitosan surface donor groups with the free binding energy $-4.44 \mathrm{kcal} / \mathrm{mol}$ (Figure $5 \mathrm{~B}$ ). It should be noted that the free binding energy of docking showed more stable testosterone-PAMAM adducts than chitosan conjugates, which is in contrast with the spectroscopic results (Figures $1 \& 2$ ).

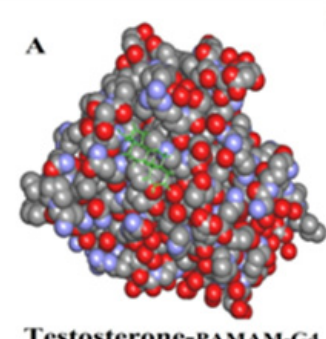
Testosterone-PAMAM- $\mathrm{C}$
AG: $--9.36 \mathrm{keal} / \mathrm{mol}$
Figure 5 B

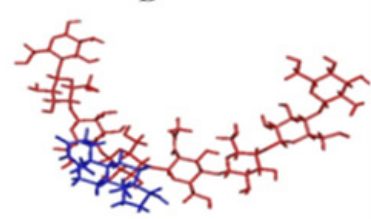

Testosterone-chitosan AG- - 4 - 4 keat
Figure 5 Best docked conformation of testosterone conjugates with PAMAM-G4 (A) and chitosan (B). View of the nearest groups surrounding testosterone (testosterone shown in green and blue color) with the free binding energy.

\section{Concluding remarks}

Testosterone forms stronger conjugate with chitosan nanoparticles than PAMAM dendrimers. Hydrophobic and H-bonding are the main 
forces in the steroid-polymer conjugation. The stability and loading efficacy of testosterone-polymer conjugates were increased as polymer size increased. Steroid encapsulation alters markedly the size and the shape of PAMAM and chitosan nanoparticles. Chitosan nanoparticles are more effective carriers for testosterone than PAMAM dendrimers.

\section{Acknowledgements}

The financial support of the Natural Sciences and Engineering Research Council of Canada (NSERC) is highly appreciated.

\section{Conflicts of interest}

None.

\section{References}

1. Samal SK, Dash M, Vlierberghe SV, et al. Cationic polymers and their therapeutic potential. Chem Soc Rev. 2012;41(21):7147-7194.

2. Svenson S. Dendrimers as versatile platform in drug delivery applications. Eur J Pharm Biopharm. 2009;71(3445-462.

3. Bernkop Schnürch A, Dünnhaupt S. Chitosan-based drug delivery systems. Eur J Pharm Biopharm. 2012;81(3):463-469.

4. Tajmir Riahi HA, Nafisi SH, Sanyakamdhorn S, et al. Applications of chitosan nanoparticles in drug delivery. Methods Mol Biol. 2014;1141:165-184.

5. Maiti PK, Caing T, Wang G, et al. Structure of PAMAM dendrimers: Generations 1 through 11. Macromolecules. 2004;37(16):6236-6254.

6. Patri AK, Majoros IJ, Baker JR. Dendritic polymer macromolecular carriers for drug delivery. Curr Opinion Chem Biol. 2002;6(4):466-471.

7. Zhao M, Hu B, Gu Z, et al. Degradable polymeric nanocapsule for efficient intracellular delivery of a high molecular weighttumorselective protein complex. Nano Today. 2013;8(1):11-20.

8. Davis ME, Chen Z, Shin DM. Nanoparticle therapeutics: an emerging treatment modality for cancer. Nat Rev Drug Discov. 2008;7(9):771-782.

9. Pacheco N, Garnica Gonzalez M, Gimeno M, et al. Structural characterization of chitin and chitosan obtained by biological and chemical methods. Biomacromolecules. 2011;12(9):3285-3290.

10. Amidi M, Mastrobattista E, Jiskoot W, et al. Chitosan-based delivery systems for protein therapeutics and antigens. Adv Drug Deliv Rev. 2010;62(1):59-82.

11. Gan Q, Wang T. Chitosan nanoparticles as protein delivery carriersystematic examination of fabrication conditions for efficient loading and release. Colloids Surf B Biointerfaces. 2007;59(1):24-34.

12. Rabea EI, Badawy MET, Stevens CV, et al. Chitosan as antimicrobial agent: application and mode of action. Biomacromolecules. 2003;4(6):1457-1465

13. Sanyakamdhorn S, Agudelo D, Tajmir Riahi HA. Encapsulation of antitumor drug doxorubicin and its analogue by chitosan nanoparticles. Biomacromolecules. 2013;14(2):557-563.

14. Agudelo D, Sanyakamdhorn S, Nafisi S, et al. Transporting antitumor drug tamoxifen and its metabolites, 4-hydroxytamoxifen and endoxifen by chitosan nanoparticles. PLoS one. 2013;8(3):e60250.

15. Bowman K, Leong KW. Chitosan nanoparticles for oral drug and gene delivery. Int J Nanomedicine. 2006;1(2):117-128.

16. Agnihotri SA, Mallikarjuna NN, Aminabhavi TM. Recent advances on chitosan-based micro- and nanoparticles in drug delivery. J Control Release. 2004;100(1):5-28.

17. Bhasin S, Woodhouse L, Casaburi R, et al. Testosterone dose-response relationships in healthy young men. Am J Physiol Endocrinol Metab. 2001;281(6):E1172-E1181.
18. Bastien D, Leblanc V, Asselin É, et al. First synthesis of separable isomeric testosterone dimers showing differential activities on prostate cancer cells. Bioorg Med Chem Lett. 2010;20(7):2078-2081.

19. Vesper AR, Lacroix J, C Gaudreault R, et al. Synthesis of novel C2symmetric testosterone dimers and evaluation of antiproliferative activity on androgen-dependent and -independent prostate cancer cell lines. Steroids. 2016;115:98-104.

20. Morin N, Bruneau J, Fortin S, et al. New testosterone derivatives as semi-synthetic anticancer agents against prostate cancer: synthesis and preliminary biological evaluation. Med Chem. 2015;11(6):531-539.

21. Bastien D, Hanna R, Leblanc V, et al. Synthesis and preliminary in vitro biological evaluation of 7a-testosterone-chlorambucil hybrid designed for the treatment of prostate cancer. Eur J Med Chem. 2013;64:442-447.

22. Fortin S, Brasseur K, Morin N, et al. New platinum(II) complexes conjugated at position $7 \mathrm{a}$ of $17 \beta$-acetyl-testosterone as new combimolecules against prostate cancer: Design, synthesis, structureactivity relationships and biological evaluation. Eur J Med Chem. 2013;68:433-443.

23. Denisov IG, Mak PJ, Grinkova YV, et al. The use of isomeric testosterone dimers to explore allosteric effects insubstrate binding to cytochrome P450 CYP3A4. J Inorg Biochem. 2016;158:77-85.

24. Chanphai P, Agudelo D, Vesper AR, et al. Effect of testosterone and its aliphatic and aromatic dimers on DNA morphology. Int $J$ Biol Macromol. 2017;95:850-855.

25. Chanphai P, Agudelo D, Vesper AR, et al. Testosterone and its dimers alter tRNA morphology. J Pharm Biomed Anal. 2017;134:269-274.

26. Chen S, Pederson D, Oak M, et al. In vivo absorption of steroidal hormones from smart polymer based delivery systems. J Pharm Sci. 2010;99(8):3381-3388.

27. Chanphai P, Vesper AR, Bekale L, et al. Transporting testosterone and its dimers by serum proteins. J Photochem Photobiol B. 2015;153:173-183.

28. Chanphai P, Bekale L, Tajmir Riahi HA. Conjugation of steroids with PAMAM nanoparticles. Colloids Surf $B$ Biointerfaces. 2015;136:1035-1041.

29. Quinones JP, Szopko R, Schmidt C, et al. Novel drug delivery systems: Chitosan conjugates covalently attached to steroids with potentialanticancer and agrochemical activity. Carbohydrate Polymers. 2011;84(3):858-864.

30. Quinones JP, Gothelf KV, Kjems J, et al. O6-partially acetylated chitosan nanoparticles hydrophobically-modified for controlled release of steroids and vitamin E. Carbohydr Polym. 2013;91(1):143-151.

31. Zhong W, Wang Y, Yu JS, et al. The interaction of human serum albumin with a novel antidiabetic agent-SU-118. J Pharm Sci. 2004;93(4):1039-1046

32. Mandeville JS, Bourassa P, Tajmir Riahi HA. Probing the binding of cationic lipids with dendrimers. Biomacromolecules. 2013;14(1):142-152.

33. Skovstrip S, Hansen SG, Skrydstrup T, et al. Conformational flexibility of chitosan: a molecular modeling study. Biomacromolecules. 2010;11(11):3196-3207.

34. Ottaviani MF, Matteini $\mathrm{P}$, Brustolon $\mathrm{M}$, et al. Characterization of starburst dendrimers and vesicle solutions and their interactions by $\mathrm{CW}$ and pulsed-EPR, TEM, and dynamic light scattering. J Phys Chem B. 1998;102(31):6029-6039.

35. Yang H, Yu D, Wang H, et al. Aggregation behavior of amphiphilic PAMAM-based hyperbranched polymer in the presence of conventional small molecular surfactants. Advances in Chemical Engineering and Science. 2013;3:11-18. 
36. Ottaviani MF, Favuzza P, Bigazzi M, et al. TEM and EPR Investigation of the competitive binding of uranyl ionsto starburst dendrimers and liposomes: potential use of dendrimers as uranyl ionsponges. Langmuir. 2000;16(19):pp;7368-7372.

37. Zhang Y, Chen J, Xiao C, et al. Cationic dendron-bearing lipids: investigating structure-activity relationships for small interfering RNA Biomacromolecules. 2013;14(12):4289-4300.

38. Tono Y, Kojima C, Haba Y, et al. Thermosensitive properties of poly(amidoamine) dendrimers with peripheral phenylalanine residues. Langmuir. 2006;22(11):4920-4922.

39. Reddy RR, Raghupathi KR, Torres DA, et al. Stimulisensitive amphiphilic dendrimers. New J Chem. 2012;36(2):340-349.

40. Liu M, Kono K, Fréchet JMJ. Water-soluble dendritic unimolecular micelles: Their potential as drug delivery agents. $J$ Control Release. 2000;65(1-2):121-131.

41. Ambade AV, Savariar EN, Thayumanavan S. Dendrimeric micelles for controlled drug release and targeted delivery. Mol Pharm. 2005;2(4):264-272.

42. Sanyakamdhorn S, Bekale L, Agudelo D, et al. Structural analysis of doxorubicin-polymer conjugates. Colloids Surf B Biointerfaces. 2015; $135: 175-182$.

43. Sanyakamdhorn S, Bekale L, Agudelo D, et al. Targeted conjugation of breast anticancer drug tamoxifen and its metabolites with synthetic polymers. Colloids and Surfaces B: Biointerfaces. 2016;145:55-63.

44. Manchanda R, Nimesh S, Controlled size chitosan nanoparticles as an efficient, biocompatible oligonucleotides delivery system. J Appl Polymer Sci. 2010;118(4):2071-2077.

45. Hembran KC, Prabha S, Chandra R, et al. Advances in preparation and characterization of chitosan nanoparticles for therapeutics. Artif Cells Nanomed Biotechnol. 2016;44(1):305-314

46. Agudelo D, Kreplak L, Tajmir Riahi HA. Microscopic and spectroscopic analysis of chitosan-DNA conjugates. Carbohydr Polym. 2015; 137:207-213.
47. Souza HKS, Gonc alves MP, Gomez J. Effect of chitosan degradation on its interaction with $\beta$-lactoglobulin. Biomacromolecules. 2011;12(4):1015-1023.

48. Sathiyabama M, Parthasarathy R. Biological preparation of chitosan nanoparticles and its in vitro antifungal efficacy against some phytopathogenic fungi. Carbohydrate Polymers. 2016;151:321-325.

49. Viveka R, Nipun Babua V, Thangama R, et al. pH-responsive drug delivery of chitosan nanoparticles as tamoxifen carriers for effective anti-tumor activity in breast cancer cells. Colloids Surf B Biointerfaces. 2013;111:117-123.

50. Zhao Y, Ma L, Zeng R, et al. Preparation, characterization and protein sorption of photo-cross linked cell membrane-mimicking chitosanbased hydrogels. Carbohydr Polym. 2016;151:237-244.

51. Hoven VP, Tangpasuthadol V, Angkitpaiboon Y, et al. Surface-charged chitosan: Preparation and protein adsorption. Carbohydrate Polymers. 200768(1):44-53.

52. Chanphai P, Tajmir Riahi HA. Conjugation of chitosan nanoparticles with biogenic and synthetic polyamines: A delivery tool for antitumor polyamine analogues. Carbohydr Polym. 2016;152:665-671.

53. Chanphai H, Tajmir Riahi HA. Thermodynamic analysis of biogenic and synthetic polyamines conjugation with PAMAM-G4 nanoparticles. J Photochem Photobiol B. 2016;155:13-19

54. Chandra S, Dietrich S, Lang H, et al. Dendrimer-doxorubicin conjugate for enhanced therapeutic effects for cancer. Journal of Materials Chemistry. 2011;21:5729-5737.

55. Popescu MC, Filip D, Vasile C, etal. Characterization by Fourier transform infrared spectroscopy (FT-IR) and 2D IR correlation spectroscopy of PAMAM dendrimer. J Phys Chem B. 2006;110(29):14198-14211.

56. Brugnerotto J, Lizardi J, Goycoole FM, et al. An infrared investigation in relation with chitin and chitosan characterization. Polymer. 2000;42(8):3569-3580. 